\title{
Testing for separability of spatial-temporal covariance functions
}

\author{
Montserrat Fuentes ${ }^{1}$
}

\section{SUMMARY}

Most applications in spatial statistics involve modeling of complex spatial temporal dependency structures, and many of the problems of space and time modeling can be overcome by using separable processes. This subclass of spatial temporal processes has several advantages, including rapid fitting and simple extensions of many techniques developed and successfully used in time series and classical geostatistics. In particular, a major advantage of these processes is that the covariance matrix for a realization can be expressed as the Kronecker product of two smaller matrices that arise separately from the temporal and purely spatial processes, and hence its determinant and inverse are easily determinable. However, these separable models are not always realistic, and there are no formal tests for separability of general spatial temporal processes. We present here a formal method to test for separability. Our approach can be also used to test for lack of stationarity of the process. The beauty of our approach is that by using spectral methods the mechanics of the test can be reduced to a simple two-factor analysis of variance (ANOVA) procedure. The approach we propose is based on only one realization of the spatial temporal process.

We apply the statistical methods proposed here to test for separability and stationarity of spatial temporal ozone fields using data provided by the US Environmental Protection Agency (EPA).

\footnotetext{
${ }^{1}$ Montserrat Fuentes is an Associate Professor at the Statistics Department, North Carolina State University (NCSU), Raleigh, NC 27695-8203, and a visiting scientist at the US Environmental Protection Agency (EPA). Tel.:(919) 515-1921, Fax: (919) 515-1169, E-mail: fuentes@stat.ncsu.edu. This research was sponsored by a National Science Foundation grant DMS 0353029 , and by a US EPA grant R-8287801.

Key words: covariance, Fourier transform, periodogram, spectral density, weakly stationarity.
} 


\section{Introduction: The need for formal methods to test for separa-}

\section{bility}

Most of the applications of spatial statistics in atmospheric sciences, ecology, oceanography, and many other sciences involve spatial temporal processes, and the difficult challenge of modeling the spatial-temporal structure can be overcome by using separable processes. A spatial-temporal field $Z(\mathbf{s}, t)$, where $\mathbf{s}$ represent space and $t$ time, is separable if $\operatorname{Cov}\left\{Z(\mathbf{s}, t), Z\left(\mathbf{s}^{\prime}, t^{\prime}\right)\right\}=C_{1}\left(\mathbf{s}, \mathbf{s}^{\prime}\right) C_{2}\left(t, t^{\prime}\right)$ for some spatial covariance $C_{1}$ and temporal covariance $C_{2}$. This class of spatial temporal processes offers enormous computational benefits, because the covariance matrix can be expressed as the Kronecker product of two smaller matrices that arise separately from the temporal and purely spatial processes, and then its determinant and inverse are easily determinable. Thus, separability is a desirable property for spatial temporal processes. However, these separable models are not always appropriate, and no formal tests are available for the hypothesis of separability of general spatial temporal processes (with a spatial component that may not be stationary). Probably, the most relevant work has been done by Guo and Billard (1998); they used the Wald test to test a doubly-geometric process against a more general unilateral autoregressive process in a time series context. For stationary spatial autoregressive processes there is an asymptotic chi-square test (Shitan and Brockwell, 1995). For "blur-generated" models, Brown et al. (2000) studied separability using a likelihood approach. Mitchell (2002), and Mitchell, Genton and Gumpertz (2002) proposed a likelihood ratio test of separability for an i.i.d. multivariate process, this likelihood-based method can be used with spatial-temporal processes that have enough replicates over time and also with multivariate repeated measures. Haas (1998) introduced a parametric Monte-Carlo hypothesis test, to study the goodness-of-fit of a particular spatial-temporal parametric model. However, there are no formal tests for more general spatial temporal models. A couple of noteworthy exceptions is the work of Scaccie and Martin (2004) and Lu and Zimmerman (2002). Scaccie and Martin (2004) introduce methods only for the spatial case (not space-time) to test axial symmetry and spatial separability using periodograms. The approach presented by Scaccie and Martin differs from the one presented here in many ways. Here we have a spatial-temporal setting and we use the coherence (rather than the un-smoothed periodogram) to test for separability. Lu and Zimmerman (2002) present a formal 
test for directional symmetry (not separability) in a spatial setting.

In this work we consider the problem of testing a given spatial temporal process for separability. The approach we propose here is based on a spectral representation of the process, and the proposed method consists essentially in studying if the coherence function of the process, is constant across frequencies. The approach we propose is based on only one realization of the spatial temporal process and it is a nonparametric test.

We also address the problem of testing for second-order stationarity of the spatial covariance of the process. This test is a side-effect of the test for separability proposed here. A random field $\mathrm{Z}$ in $\mathbb{R}^{2}$ is called weakly stationary (or stationary), if it has finite second moments, its mean function is constant and it possesses an autocovariance function $C$, such that $C(\mathbf{x}-\mathbf{y})=\operatorname{cov}\{Z(\mathbf{x}), Z(\mathbf{y})\}$. The conventional methods in spatial statistics are essentially based on the assumption of stationary random fields. However, such assumptions might not hold in large heterogeneous fields (Fuentes, 2001, 2002). Most of the work that has been done so far to test the nonstationarity of a random process is for time series. Several authors have proposed methods for testing whether or not a given time-series may be regarded as stationary, some of these methods are designed to detect nonstationary "trends". Priestley and Rao (1969) used the concept of the "evolutionary (time-dependent) spectrum" to test the lack of stationarity of a time series. Unfortunately, the literature does not offer many approaches to test for non-stationarity of spatial processes. A couple of noteworthy exceptions are Bose and Steinhardth (1996) and Ephraty, Tabrikian and Messer (2001). The test by Bose et al. is sensitive to the centrosymmetry property of the sample covariance matrix of a spatial process sampled using a uniform, linear array. Our approach is not sensitive to the centrosymmetry property, since we use spectral methods rather than covariance structures. The method of Ephraty et al. relies on empirical spectral methods to test a low degree of stationarity. This low degree of stationarity is arbitrary fixed. Ephraty et al. also introduce a likelihood-based approach to test for stationarity. Our approach is not likelihood-based. Fuentes (2003) proposed a method to test stationarity of a spatial process using the spatial periodogram for lattice data. The method to be described in this paper may be used to test the stationarity of the second-order properties of the spatial covariance of a space-time process, it is based on the coherency function and it does not require gridded data. 
This paper is organized as follows. In Section 2 we introduce a spectral interpretation of separability. In Section 3 we present a nonparametric estimate of the spectrum of a spatial-temporal process and we study its asymptotic properties. In Section 4 we introduce a novel approach to test for separability and stationarity. In Section 5 we apply the statistical methods proposed here to a study of the separability and stationarity of ozone fields. Section 6 is dedicated to a study of the power of the test.

\section{Spectral interpretation of separability}

\subsection{Separable stationary processes}

In this section we introduce a spectral analog of the assumption of separability, assuming stationarity in space and time for the covariance function. We will relax this assumption in Section 2.2, where we will extend the approach presented here to processes with a nonstationary spatial covariance.

Consider $\left\{Z(\mathbf{s} ; t): \mathbf{s} \in D \subset \mathbb{R}^{d} ; t \in \mathbb{Z}\right\}$ a spatial temporal zero-mean process observed at $N$ space-time coordinates $\left(\mathbf{s}_{1} ; t_{1}\right), \ldots,\left(\mathbf{s}_{N} ; t_{N}\right)$. We start by assuming that the covariance function is stationary in space and time,

$$
\operatorname{cov}\left(Z\left(\mathbf{s}_{1} ; t_{1}\right), Z\left(\mathbf{s}_{2} ; t_{2}\right)\right)=C(\mathbf{h} ; u)
$$

for $\mathbf{h}=\mathbf{s}_{1}-\mathbf{s}_{2}, u=t_{1}-t_{2}$.

By Bochner's theorem we can write the covariance $C$ in terms of the spectral density $g$ of the spatial temporal process $Z$,

$$
C(\mathbf{h}, u)=\iint \exp \left\{i \mathbf{h}^{T} \boldsymbol{\omega}+i u \tau\right\} g(\boldsymbol{\omega} ; \tau) d \boldsymbol{\omega} d \tau
$$

If $C$ is integrable, then we have

$$
\begin{aligned}
g(\boldsymbol{\omega}, \tau) & =(2 \pi)^{-d-1} \sum_{u=-\infty}^{u=\infty} \int \exp \left\{-i \mathbf{h}^{T} \boldsymbol{\omega}-i u \tau\right\} C(\mathbf{h} ; u) d \mathbf{h} \\
& =(2 \pi)^{-d} \int \exp \left\{-i \mathbf{h}^{T} \boldsymbol{\omega}\right\} f(\mathbf{h} ; \tau) \mathbf{d h}
\end{aligned}
$$

where for any fixed $\mathbf{h}, f(\mathbf{h} ; \tau)$ is the cross-spectral density function of the time processes $Y_{1}(t)=Z(\mathbf{s} ; t)$ and $Y_{2}(t)=Z(\mathbf{s}+\mathbf{h} ; t)$, and we have, 


$$
f(\mathbf{h} ; \tau)=(2 \pi)^{-1} \sum_{u=-\infty}^{u=\infty} \exp \{-i u \tau\} C(\mathbf{h}, u) .
$$

If $C$ is a separable covariance then we can write

$$
C(\mathbf{h}, u)=C_{1}(\mathbf{h}) C_{2}(u)
$$

where $C_{1}$ is a positive-definite function in $\mathbb{R}^{d}$, and $C_{2}$ is a positive-definite function in $\mathbb{R}$. Thus, $f(\mathbf{h} ; \tau)$ is the product of a function of $\mathbf{h}$ and a function of $\tau$,

$$
f(\mathbf{h} ; \tau)=(2 \pi)^{-1} \sum_{u=-\infty}^{u=\infty} \exp \{-i u \tau\} C(\mathbf{h}, u)=(2 \pi)^{-1} \sum_{u=-\infty}^{u=\infty} \exp \{-i u \tau\} C_{1}(\mathbf{h}) C_{2}(u)=C_{1}(\mathbf{h}) \kappa(\tau)
$$

where $\kappa$ is an integrable and positive function, and $C_{1}$ for each fixed $\tau$ is a covariance function of $\mathbf{h}$ and an integrable function of $\mathbf{h}$. We can obtain a nonseparable covariance function by making $C_{1}$ depend on $\tau$. Thus, we get

$$
C(\mathbf{h}, u)=\int \exp \{i u \tau\} C_{1}(\mathbf{h} ; \tau) \kappa(\tau) \mathbf{d} \tau .
$$

Cressie and Huang (1999) use this spectral representation to generate parametric models of nonseparable spatio-temporal stationary covariance functions.

Thus, if $Z$ is separable,

$$
f(\mathbf{h} ; \tau)=C_{1}(\mathbf{h}) \kappa(\tau)
$$

In principle $f$ could be a complex function, but when $Z$ is separable and stationary $f$ is real.

\subsection{Nonstationarity in space}

In this Section we present the spectral analog of separability without assuming the process is second-order stationary in space. Thus, if $Z$ is not second order stationary in space, the cross-spectral function $f(\cdot ; \tau)$ between the two time processes $X_{1}(t)=Z(\mathbf{a} ; t)$ and $X_{2}(t)=Z(\mathbf{b} ; t)$, is not just a function of the spatial distance between two points $\mathbf{a}$ and $\mathbf{b}$ in $D$, but it depends also on the location of $\mathbf{a}$ and $\mathbf{b}$. Then, we write the cross-spectral function as $f_{\mathbf{a}, \mathbf{b}}(\tau)$, for $\mathbf{a}$ and $\mathbf{b}$ in $D$, and $\tau \in[0, \infty)$,

$$
f_{\mathbf{a}, \mathbf{b}}(\tau)=(2 \pi)^{-1} \sum_{u=-\infty}^{u=\infty} \exp \{-i u \tau\} \operatorname{cov}\{Z(\mathbf{a} ; u+t), Z(\mathbf{b} ; t)\}
$$


We define now the coherency between site $\mathbf{a}$ and $\mathbf{b}$ :

$$
R_{\mathbf{a b}}(\tau)=\frac{\left|f_{\mathbf{a b}}(\tau)\right|}{\left[f_{\mathbf{a a}}(\tau) f_{\mathbf{b b}}(\tau)\right]^{1 / 2}},
$$

$\left|R_{\mathbf{a b}}(\tau)\right|^{2}$ is the frequency analog of the coefficient of correlation between the two time processes $X_{1}(t)=$ $Z(\mathbf{a} ; t)$ and $X_{2}(t)=Z(\mathbf{b} ; t)$. If the process has a separable covariance nonstationary in space (stationary in time), we have

$$
\operatorname{cov}\{Z(\mathbf{a} ; u+t), Z(\mathbf{b} ; t)\}=C_{s}(\mathbf{a}, \mathbf{b}) C_{2}(u)
$$

where $C_{s}$ is a spatial covariance, and then,

$$
f_{\mathbf{a b}}(\tau)=C_{s}(\mathbf{a}, \mathbf{b}) \kappa(\tau)
$$

where $\kappa$ is a positive function. Therefore,

$$
R_{\mathbf{a b}}(\tau)=\frac{\left|f_{\mathbf{a b}}(\tau)\right|}{\left[f_{\mathbf{a a}}(\tau) f_{\mathbf{b b}}(\tau)\right]^{1 / 2}}=\frac{C_{s}(\mathbf{a}, \mathbf{b})}{\left[C_{s}(\mathbf{a}, \mathbf{a}) C_{s}(\mathbf{b}, \mathbf{b})\right]^{1 / 2}}
$$

Thus, if the process is separable, the coherency, $R_{\mathbf{a b}}(\tau)$, does not depend on the frequency $\tau$. Furthermore, if the process is stationary in space, the covariance function $C_{s}$ is stationary, and then $R_{\mathbf{a b}}(\tau)$ depends on $\mathbf{a}, \mathbf{b}$ only through the vector distance between the two locations.

We test for separability by studying if $R_{\mathrm{ab}}(\tau)$ depends on the frequency $\tau$. The stationary test is a side-effect test, we test for stationarity by studying if $R_{\mathbf{a}_{i} \mathbf{b}_{i}}(\tau)$ depends on location, when $R$ is evaluated (estimated) at pairs $\left\{\left(\mathbf{a}_{i}, \mathbf{b}_{i}\right)\right\}_{i}$ all separated by the same distance $\mathbf{h}$. In the next section we present an estimate of $R$.

\section{Spectral estimates}

\subsection{Estimating the spectral density}

We propose in this section a nonparametric estimate of the cross-spectral density $f_{\mathbf{a b}}(\omega)$ and we study its asymptotic properties (as $T \rightarrow \infty$ ). Our estimate, $\hat{f}_{\mathbf{a b}}(\omega)$, is a spatial and temporal smoothed version of the cross-periodogram for the two time processes $Z(\mathbf{a} ; t)$ and $Z(\mathbf{b} ; t)$. We use two filters; a spectral filter $W$, and a spatial filter $g_{\rho}$, to obtain a weighted average of the cross-periodogram values concentrating weight in a neighborhood of $\omega$ (filter $W$ ), and in a spatial neighborhood of $\mathbf{a}$ and $\mathbf{b}$ (filter $g_{\rho}$ ). 
We first define $J_{\mathbf{a}}(\omega)$,

$$
J_{\mathbf{a}}(\boldsymbol{\omega})=\sum_{t=0}^{T-1} K(t / T) Z(\mathbf{a}, t) \exp \{-i t \omega\}
$$

where $T=t_{N}$. We define the cross-periodogram $I_{\mathbf{a b}}(\omega)$,

$$
I_{\mathbf{a b}}(\omega)=\left\{2 \pi \sum_{t=0}^{T-1} K(t / T)^{2}\right\}^{-1} J_{\mathbf{a}}(\omega) J_{\mathbf{b}}^{c}(\omega),
$$

where $K$ is a tapering function. The entries of $I_{\mathbf{a b}}(\omega)$ are the second order periodogram of the tapered values $K(t / T) Z(\mathbf{a}, t)$ for $t=0, \pm 1, \ldots$ We shall form a consistent estimate of $f_{\mathbf{a b}}$ by taking a weighted average of the statistic $I_{\mathbf{a b}}(\omega)$ concentrating weight in a neighborhood of $\omega$ having width $O(B)$ where $B$ is a band-width parameter tending to 0 as $T \rightarrow \infty$. Let $W(\alpha),-\infty<\alpha<\infty$, be a weight function satisfying

$$
\int_{-\infty}^{\infty} W(\alpha) d \alpha=1
$$

Let $B, T=1,2, \ldots$ be a bounded sequence of non-negative scale parameters. We consider

$$
I_{\mathbf{a b}}^{*}(\omega)=2 \pi / T \sum_{t=0}^{T-1} W^{(T)}(\omega-2 \pi t / T) I_{\mathbf{a b}}(2 \pi t / T),
$$

where

$$
W^{(T)}(\alpha)=B^{-1} \sum_{j=-\infty}^{\infty} W\left(B^{-1}[\alpha+2 \pi j]\right) .
$$

The estimate (9) weights periodogram values heavily at frequencies within $O(B)$ of $\omega$. This suggests that we will later require $B \rightarrow 0$ as $T \rightarrow \infty$. We estimate $f_{\mathbf{a b}}(\omega)$ by "smoothing" the values of $I_{\mathbf{a b}}^{*}(\omega)$ over neighboring values of $\mathbf{a}$, and $\mathbf{b}$. More precisely, let $g_{\rho}$ be a weight function or "window", depending on the bandwidth parameter $\rho$, which satisfies

C.1 $g_{\rho}(\mathbf{s}) \geq 0$, for all $\mathbf{s}, \rho$.

C.2 $g_{\rho}(\mathbf{s})$ decays to zero as $|\mathbf{s}| \rightarrow \infty$, for all $\rho$.

\section{C.3}

$$
\int_{-\infty}^{+\infty} \int_{-\infty}^{+\infty} g_{\rho}(\mathbf{s}) d \mathbf{s}=1, \quad \text { for all } \rho
$$

\section{C.4}

$$
\int_{-\infty}^{+\infty} \int_{-\infty}^{+\infty}\left\{g_{\rho}(\mathbf{s})\right\}^{2} d \mathbf{s}<\infty, \quad \text { for all } \rho
$$


Then, we estimate $f_{\mathbf{a b}}(\omega)$ with

$$
\hat{f}_{\mathbf{a b}}(\omega)=\int_{-\infty}^{\infty} \int_{-\infty}^{\infty} g_{\rho}(\mathbf{a}-\mathbf{s}) g_{\rho}(\mathbf{b}-\mathbf{s}) I_{\mathbf{a}+\mathbf{s}, \mathbf{b}+\mathbf{s}}^{*}(\omega) d \mathbf{s}
$$

Thus, $\hat{f}_{\mathbf{a b}}(\omega)$ can be interpreted as an average of the total energy of the process contained within a band of frequencies in the region of $\omega$ and a region in space in the neighborhood of $\mathbf{a}$, and $\mathbf{b}$.

\section{Examples of filters:}

In the application presented in this article we consider $\left\{g_{\rho}(\mathbf{s})\right\}$ for $\mathbf{s}=\left(s_{1}, s_{2}\right)$ to be a multiplicative filter, i.e. the tensor product of two 1-dimensional filters, $g_{\rho}(\mathbf{s})=g_{1}\left(s_{1}\right) g_{1}\left(s_{2}\right)$, where $g_{1}$ is of the form

$$
g_{1}(s)= \begin{cases}1 / \rho & (-1 / 2) \rho \leq s \leq(1 / 2) \rho \\ 0 & \text { otherwise }\end{cases}
$$

corresponding to the Daniell window. In the application we also consider the following weight function,

$$
W_{\mathbf{a b}}^{(T)}\left(\frac{2 \pi s}{T}\right)=\frac{T}{2 \pi}(2 m+1)^{-1}
$$

with $m=B T$, and $s \leq m$. This is a constant function; it is the weight of the $2 B T+1$ periodogram ordinates whose frequencies fall in the interval $(\lambda-2 \pi B, \lambda+2 \pi B)$. Thus,

$$
I_{\mathbf{a b}}^{*}(\lambda)=(2 m+1)^{-1} \sum_{j=-m}^{m} I_{\mathbf{a b}}\left(\frac{2 \pi[s(T)+j]}{T}\right)
$$

where $s(T)$ an integer with $2 \pi s(T) / T$ near $\lambda$.

The tapering function $K$ in the application presented in this paper is considered constant, i.e. $K(\mathbf{x})=1$, for all $\mathbf{x}$.

Asymptotic distribution of the estimated spectrum:

We define $\hat{f}_{D}(\omega)$ the matrix with entries $\hat{f}_{\mathbf{a b}}(\omega)$ for all pairs $\{\mathbf{a}, \mathbf{b}\}$ in our domain. Next, we study the asymptotic properties and distribution of $\hat{f}_{D}\left(\omega_{1}\right), \ldots, \hat{f}_{D}\left(\omega_{J}\right)$.

We introduce first some assumptions:

A.1 The weight function $W(\beta),-\infty<\beta<\infty$, is real-valued, even, and of bounded variation

$$
\int_{-\infty}^{\infty} W(\beta) d \beta=1
$$


and

$$
\int_{-\infty}^{\infty}|W(\beta)| d \beta<\infty
$$

A.2 The temporal covariance is summable, then for each $\mathbf{h}$,

$$
\sum_{u=-\infty}^{u=\infty}|C(\mathbf{h}, u)|<\infty
$$

and also

$$
\sum_{u=-\infty}^{u=\infty}|u||C(\mathbf{h}, u)|<\infty
$$

A.3 We assume $T \rightarrow \infty, B T \rightarrow \infty$, and $B \rightarrow 0$.

\section{Theorem 1}

Consider a Gaussian spatial temporal process $\left\{Z(\mathbf{s} ; t): \mathbf{s} \in D \subset \mathbb{R}^{d} ; t \in \mathbb{Z}\right\}$, observed at $N$ space-time coordinates $\left(\mathbf{s}_{1} ; t_{1}\right), \ldots,\left(\mathbf{s}_{N} ; t_{N}\right)$, and with a covariance function $\operatorname{cov}\left(Z\left(\mathbf{s}_{1}, t_{1}\right), Z\left(\mathbf{s}_{2}, t_{2}\right)\right)=C\left(\mathbf{s}_{1}-\mathbf{s}_{2}, t_{1}-t_{2}\right)$. We define for $\omega \in[-\pi, \pi]$ a second order periodogram function, $\hat{f}_{\mathbf{a b}}(\omega)$ for all pairs $\{\mathbf{a}, \mathbf{b}\}$ in $D$, as an estimate of the cross-spectral function $f_{\mathbf{a b}}(\omega)$ defined in (5). We consider two filters $\left\{g_{\rho}(\mathbf{s})\right\}$ (satisfying C.1-C.4) and $\{W(\beta)\}$.

Under assumptions A.1, A.2 and A.3, we get:

- (i) The expected of value of the estimated spectrum $\hat{f}_{\mathbf{a b}}$, is

$$
\begin{aligned}
E\left(\hat{f}_{\mathbf{a b}}(\omega)\right) & =\frac{2 \pi}{T} \sum_{s=1}^{T-1} W^{(T)}\left(\omega-\frac{2 \pi s}{T}\right) \tilde{f}_{\mathbf{a b}}\left(\frac{2 \pi s}{T}\right)+O\left(T^{-1}\right) \\
& =\int_{-\infty}^{\infty} W(\alpha) \tilde{f}_{\mathbf{a b}}(\omega-B \alpha) d \alpha+O\left(B^{-1} T^{-1}\right)
\end{aligned}
$$

where the error term is uniform in $\omega$, and

$$
\tilde{f}_{\mathbf{a b}}(\omega)=\int_{-\infty}^{\infty} \int_{-\infty}^{\infty} g_{\rho}(\mathbf{a}-\mathbf{s}) g_{\rho}(\mathbf{b}-\mathbf{s}) f_{\mathbf{a}+\mathbf{s}, \mathbf{b}+\mathbf{s}}(\omega) d \mathbf{s}
$$

for $-\infty<\omega<\infty$.

- (ii) The asymptotic variance of $\hat{f}_{\mathbf{a b}}$, is

$$
\begin{aligned}
\operatorname{cov}\left\{\hat{f}_{\mathbf{a}_{1}, \mathbf{b}_{1}}(\lambda), \hat{f}_{\mathbf{a}_{2}, \mathbf{b}_{2}}(\omega)\right\} & =\frac{2 \pi}{T}\left[\int_{-\pi}^{\pi} W^{(T)}(\lambda-\alpha) W^{(T)}(\omega-\alpha)\left[\tilde{f}_{\mathbf{a}_{1} \mathbf{a}_{2}}(\alpha) * \tilde{f}_{\mathbf{b}_{1} \mathbf{b}_{2}}(-\alpha)\right] d \alpha\right. \\
& \left.+\int_{-\pi}^{\pi} W^{(T)}(\lambda-\alpha) W^{(T)}(\omega+\alpha)\left[\tilde{f}_{\mathbf{a}_{1} \mathbf{b}_{2}}(\alpha) * \tilde{f}_{\mathbf{b}_{1} \mathbf{a}_{2}}(-\alpha)\right] d \alpha\right] \\
& +O\left(B^{-2} T^{-2}\right)+O\left(T^{-1}\right),
\end{aligned}
$$


where

$\left[\tilde{f}_{\mathbf{a}_{i} \mathbf{b}_{i}}(\lambda) * \tilde{f}_{\mathbf{a}_{j} \mathbf{b}_{j}}(\omega)\right]=\int_{\mathbb{R}^{2}} \int_{\mathbb{R}^{2}} f_{\mathbf{a}_{i}+\mathbf{s}_{i}, \mathbf{b}_{i}+\mathbf{s}_{i}}(\lambda) f_{\mathbf{a}_{j}+\mathbf{s}_{j}, \mathbf{b}_{j}+\mathbf{s}_{j}}(\omega)\left\{g_{\rho}\left(\mathbf{a}_{i}-\mathbf{s}_{i}\right) g_{\rho}\left(\mathbf{a}_{j}-\mathbf{s}_{j}\right) g_{\rho}\left(\mathbf{b}_{i}-\mathbf{s}_{i}\right) g_{\rho}\left(\mathbf{b}_{j}-\mathbf{s}_{j}\right)\right\} d \mathbf{s}_{i} d \mathbf{s}_{j}$

The error terms in (14) is uniform in $\lambda, \omega$.

- (iii) The spatial periodogram values for $\hat{f}_{\mathbf{a}_{1} \mathbf{b}_{2}}(\omega)$ and $\hat{f}_{\mathbf{a}_{2} \mathbf{b}_{2}}(\lambda)$ are asymptotically uncorrelated, for $\omega \pm \lambda \neq 0(\bmod 2 \pi)$. We have,

$$
\begin{aligned}
\lim _{T \rightarrow \infty} B T \operatorname{cov}\left\{\hat{f}_{\mathbf{a}_{1} \mathbf{b}_{1}}(\lambda), \hat{f}_{\mathbf{a}_{2} \mathbf{b}_{2}}(\omega)\right\}=2 \pi\left(\int W(\alpha)^{2} d \alpha\right) \\
\times\left(\eta(\lambda-\omega)\left[\tilde{f}_{\mathbf{a}_{1} \mathbf{a}_{2}}(\lambda) * \tilde{f}_{\mathbf{b}_{1} \mathbf{b}_{2}}(-\lambda)\right]+\eta(\lambda+\omega)\left[\tilde{f}_{\mathbf{a}_{1} \mathbf{b}_{2}}(\lambda) * \tilde{f}_{\mathbf{a}_{2} \mathbf{b}_{1}}(-\lambda)\right]\right)
\end{aligned}
$$

where

$$
\eta\{\lambda\}=\left\{\begin{array}{cc}
1 & \text { if } \lambda \equiv 0(\bmod 2 \pi) \\
0 & \text { otherwise }
\end{array}\right.
$$

- (iv) $\hat{f}_{D}\left(\omega_{1}\right), \ldots, \hat{f}_{D}\left(\omega_{J}\right)$ are asymptotically jointly normal distributed with covariance structure given by (14).

The proofs for Theorem 1 are in the appendix. By Theorem 1 we obtain the asymptotic normality and independence of $\hat{f}_{\mathbf{a}_{1} \mathbf{b}_{1}}(\omega)$ evaluated at different frequencies and locations. If we define the distance between pairs $\left(\mathbf{a}_{i}, \mathbf{b}_{i}\right)$ and $\left(\mathbf{a}_{j}, \mathbf{b}_{j}\right)$ as the minimum distance between any of the two points in the first pair and any of the two points in the second pair. Then, the spatial periodogram values for $\hat{f}_{\mathbf{a}_{1} \mathbf{b}_{2}}(\omega)$ and $\hat{f}_{\mathbf{a}_{2} \mathbf{b}_{2}}(\lambda)$ by $(14)$, are approximately independent if either:

$\|\omega \pm \lambda\|$ is sufficiently large so that

$$
\int_{-\infty}^{\infty} \int_{-\infty}^{\infty}|W(\theta+\omega)|^{2}|W(\theta+\lambda)|^{2} d \theta=0
$$

i.e. if $\|\omega \pm \lambda\| \gg$ bandwidth of $|W(\theta)|^{2}$ or the distance between pairs $\left(\mathbf{a}_{i}, \mathbf{b}_{i}\right)$ and $\left(\mathbf{a}_{j}, \mathbf{b}_{j}\right)$ is greater than the bandwidth of the function $\left\{g_{\rho}(\mathbf{u})\right\}$.

Thus, in practice, we can make the covariance in (14) almost zero by having the frequencies $\omega$ and $\lambda$ and the pairs $\left(\mathbf{a}_{1}, \mathbf{b}_{1}\right)$ and $\left(\mathbf{a}_{2}, \mathbf{b}_{2}\right)$ sufficiently apart. 


\subsection{Estimating the Coherency}

In this section we propose an estimate for the coherency function, as defined in (6), and we study its asymptotic properties.

The coherency is usually estimated by replacing $f$ with the second-order periodogram. Here we propose to estimate $R$ with

$$
\hat{R}_{\mathbf{a b}}(\tau)=\frac{\left|\hat{f}_{\mathbf{a b}}(\tau)\right|}{\left[\hat{f}_{\mathbf{a a}}(\tau) \hat{f}_{\mathbf{b b}}(\tau)\right]^{1 / 2}},
$$

where $\hat{f}$ is the proposed estimate (10) of the cross-temporal spectrum.

We define $\hat{R}_{D}(\tau)$ the matrix with entries $\hat{R}_{\mathbf{a b}}(\tau)$ for all pairs $\{\mathbf{a}, \mathbf{b}\}$ in our spatial domain. In Theorem 2, we study the asymptotic distribution of $\hat{R}_{D}\left(\tau_{1}\right), \ldots, \hat{R}_{D}\left(\tau_{J}\right)$, and the asymptotic moments of $\left|\hat{R}_{\mathrm{ab}}(\tau)\right|^{2}$. The reason to study $\left|\hat{R}_{\mathbf{a b}}(\tau)\right|^{2}$ in Theorem 2, it is because the moments of this function will help us to determine the variance stabilizing transformation of $\hat{R}_{\mathbf{a b}}(\tau)$. In Corollary 2 we present the asymptotic properties of $\tanh ^{-1}\left(\hat{R}_{\mathbf{a}, \mathbf{b}}(\tau)\right.$, which is the proposed variance stabilizing transformation of the coherency.

Theorem 2. Under assumptions A.1-A.3 and C.1-C.4.

(i) The variates $\hat{R}_{D}\left(\tau_{j}\right)$ are asymptotically jointly normal for $j=1, \ldots, J$.

$$
E\left\{\left|\hat{R}_{\mathbf{a b}}(\tau)\right|^{2}\right\}=\left|\tilde{R}_{\mathbf{a b}}(\tau)\right|^{2}+O(B)+O\left(B^{-1} T^{-1}\right)
$$

where

$$
\begin{gathered}
\tilde{R}_{\mathbf{a b}}(\tau)=\frac{\left|\tilde{f}_{\mathbf{a b}}(\tau)\right|}{\left[\tilde{f}_{\mathbf{a a}}(\tau) \tilde{f}_{\mathbf{b b}}(\tau)\right]^{1 / 2}} . \\
\operatorname{cov}\left\{\left|\hat{R}_{\mathbf{a b}}(\tau)\right|^{2},\left|\hat{R}_{\mathbf{a b}}(\lambda)\right|^{2}\right\}=[\eta\{\tau-\lambda\}+\eta\{\tau+\lambda\}]\left|\tilde{R}_{\mathbf{a b}}(\tau)\right|^{2} \\
\times\left[1-\left|\tilde{R}_{\mathbf{a b}}(\tau)\right|^{2}\right]^{2} 4 \pi \int W(\alpha)^{2} d \alpha B^{-1} T^{-1}+O\left(B^{-2} T^{-2}\right) .
\end{gathered}
$$

An examination of expression (20) with the second moments for $\left|\hat{R}_{\mathbf{a b}}(\tau)\right|^{2}$, suggests the consideration of the inverse hyperbolic tangent for $\hat{R}$ as a variance stabilizing transformation (Rao, 1973, p. 432). Thus, we 
consider the standardized asymptotic distribution of the variance stabilizing transformation of $R$, i.e.

$$
\phi_{\mathbf{a}, \mathbf{b}}(\tau)=\tanh ^{-1}\left(\tilde{R}_{\mathbf{a}, \mathbf{b}}(\tau)\right)
$$

and we estimate it with

$$
\hat{\phi}_{\mathbf{a}, \mathbf{b}}(\tau)=\tanh ^{-1}\left(\hat{R}_{\mathbf{a}, \mathbf{b}}(\tau)\right)
$$

Kendall and Stuart (1966) suggests that the transformed variate may be more nearly normal than the untransformed one. This is a straightforward consequence of the delta method (Mann and Wald, 1943).

We write $\hat{\phi}_{D}(\tau)$ to denote the matrix with entries $\hat{\phi}_{\mathbf{a b}}(\tau)$ for all pairs $\{\mathbf{a}, \mathbf{b}\}$. We study the asymptotic properties and distribution of $\hat{\phi}_{D}\left(\tau_{1}\right), \ldots, \hat{\phi}_{D}\left(\tau_{J}\right)$.

Corollary 2 Under assumptions A.1-A.3 and C.1-C.4, and if the temporal spectrum, $f_{D}(\tau)$, is nonsingular. Then,

(i) The variates $\hat{\phi}_{D}\left(\tau_{j}\right)$ are asymptotically jointly normal for $j=1, \ldots, J$.

$$
\begin{gathered}
E\left\{\hat{\phi}_{\mathbf{a b}}(\tau)\right\}=\phi_{\mathbf{a b}}(\tau)+O(B)+O\left(B^{-1} T^{-1}\right) \\
\operatorname{cov}\left\{\hat{\phi}_{\mathbf{a b}}(\tau), \hat{\phi}_{\mathbf{a b}}(\lambda)\right\}=[\eta\{\tau-\lambda\}+\eta\{\tau+\lambda\}] B^{-1} T^{-1} \pi \int W(\alpha)^{2} d \alpha \\
+O\left(B^{-2} T^{-2}\right)
\end{gathered}
$$

The results in this section show that $\hat{\phi}_{\mathbf{a b}}(\tau)$ evaluated at different locations and different frequencies can be treated approximately as a jointly distributed normal variable with constant variance and independent components.

\section{Testing for separability}

This section is the main contribution of this paper. We propose a test for separability of spatial-temporal processes. The beauty of this method is that the mechanics of the test can be reduced to those of a simple two-way ANOVA procedure. We test for separability by studying if $\hat{\phi}_{\mathbf{a b}}(\tau)$ is a function of $\tau$. 
We evaluate $\hat{\phi}_{\left(\mathbf{a}_{i}, \mathbf{b}_{i}\right)}(\tau)$, at $k$ pairs at pairs of locations $\left\{\left(\mathbf{a}_{i}, \mathbf{b}_{i}\right)\right\}_{i=1}^{k}$ and a set of frequencies $\tau_{1}, \tau_{2}, \ldots, \tau_{n}$ that cover the domain. We write

$$
\hat{\phi}_{\left(\mathbf{a}_{i}, \mathbf{b}_{i}\right)}\left(\tau_{j}\right)=\phi_{\left(\mathbf{a}_{i}, \mathbf{b}_{i}\right)}\left(\tau_{j}\right)+\epsilon\left(\left(\mathbf{a}_{i}, \mathbf{b}_{i}\right), \tau_{j}\right) .
$$

Asymptotically $E\left\{\epsilon\left(\left(\mathbf{a}_{i}, \mathbf{b}_{i}\right), \tau_{j}\right)\right\}=0$ and $\operatorname{Var}\left\{\epsilon\left(\left(\mathbf{a}_{i}, \mathbf{b}_{i}\right), \tau_{j}\right)\right\}=\sigma^{2}$ where $\sigma^{2}$ is independent of $\left(\mathbf{a}_{i}, \mathbf{b}_{i}\right)$, and $\omega_{j}$.

Assuming the $\left(\mathbf{a}_{i}, \mathbf{b}_{i}\right)$ and $\tau_{j}$ are spaced "sufficiently wide apart," then the $\epsilon\left(\left(\mathbf{a}_{i}, \mathbf{b}_{i}\right), \tau_{j}\right)$ will be approximately uncorrelated, this is based on the asymptotic properties of $\hat{f}_{\left(\mathbf{a}_{i} \mathbf{b}_{i}\right)}\left(\tau_{j}\right)$ (Theorem 1 (iii)). We write

$$
\begin{aligned}
& U_{i j}=\hat{\phi}_{\left(\mathbf{a}_{i}, \mathbf{b}_{i}\right)}\left(\tau_{j}\right), \\
& m_{i j}=\phi_{\left(\mathbf{a}_{i}, \mathbf{b}_{i}\right)}\left(\tau_{j}\right),
\end{aligned}
$$

and

$$
\epsilon_{i j}=\epsilon\left(\left(\mathbf{a}_{i}, \mathbf{b}_{i}\right), \tau_{j}\right)
$$

Then, we have the model

$$
U_{i j}=m_{i j}+\epsilon_{i j}
$$

that becomes the usual "two-factor analysis of variance" model, and can be rewritten:

$$
H_{1}: U_{i j}=\mu+\alpha_{i}+\beta_{j}+\epsilon_{i j}
$$

for $i=1, \ldots, k$ and $j=1, \ldots, n$. The parameters $\left\{\alpha_{i}\right\},\left\{\beta_{j}\right\}$ represent the main effects of the space and frequency factors. Then, we test for separability by using the standard techniques to test the model $\left(\beta_{j}=0\right)$ :

$$
H_{0 a}: U_{i j}=\mu+\alpha_{i}+\epsilon_{i j}
$$

against the more general model $H_{1}$.

\subsection{Testing for stationarity}

Much of the original impetus for spatial methods was driven by geostatistics. However, the methodology in geostatistics is essentially based on the assumption of second-order stationary random fields. Such assumption 
might not hold in large heterogeneous fields. Thus, it is important to understand when stationarity is a reasonable assumption. In this section we propose a new test for spatial stationarity.

We test for spatial stationarity by studying if the coherency, $R$, is a function of space, when $R$ is evaluated at pairs of locations $\left\{\left(\mathbf{a}_{i}, \mathbf{b}_{i}\right)\right\}_{i=1}^{k}$ separated by the same vector distance $\mathbf{h}$, i.e. $\mathbf{a}_{i}-\mathbf{b}_{i}=\mathbf{h}$ for all $i=1, \ldots, k$.

If the process is stationary in space, at each fixed time the covariance of the process between two locations $\mathbf{a}$ and $\mathbf{b}$ separated by a distance $\mathbf{h}$, is only a function of $\mathbf{h}$. Thus, the spectral function $f_{\mathbf{a b}}(\tau)$ is only a function of $\tau$ and $\mathbf{h}$, and we denote it with $f(\mathbf{h}, \tau)$ as in (3). Then, the coherency is just a function of vector distances between pair of locations:

$$
R(\mathbf{h} ; \tau)=|f(\mathbf{h} ; \tau)| /|f(\mathbf{0} ; \tau)|
$$

Furthermore, under the assumption of separability (see Eq. 4), we have

$$
R(\mathbf{h} ; \tau)=|f(\mathbf{h} ; \tau)| /|f(\mathbf{0} ; \tau)|=C_{1}(\mathbf{h}) / C_{1}(\mathbf{0})
$$

We define $R_{i j}$ as in (23), but we evaluate it only at pairs $\left\{\left(\mathbf{a}_{i}, \mathbf{b}_{i}\right)\right\}_{i=1}^{k}$, such that $\mathbf{a}_{i}-\mathbf{b}_{i}=\mathbf{h}$ for all $i=1, \ldots, k$. We test for stationarity in space by using the standard ANOVA techniques to test the model $\left(\alpha_{i}=0\right)$ :

$$
H_{0 b}: R_{i j}=\mu+\beta_{j}+\epsilon_{i j}
$$

against a more general model $H_{1}$,

$$
H_{1}: R_{i j}=\mu+\alpha_{i}+\beta_{j}+\epsilon_{i j}
$$

for $i=1, \ldots, k$ and $j=1, \ldots, n$ where parameters $\left\{\alpha_{i}\right\},\left\{\beta_{j}\right\}$ represent the main effects of the space and frequency factors. We can also test for separability by studying if the frequency effect is zero $\left(\beta_{j}=0\right)$.

\section{Application}

Our goal is to understand the spatial temporal structure of air pollutants using the output of the regional scale air quality models (Models-3). Models-3 estimates hourly concentrations and fluxes of different pollutants. The primary objective of Models-3 is to improve the environmental management community's ability to evaluate the impact of air quality management practices for multiple pollutants at multiple scales, as part 
of the regulation process of the air pollutants standards. As an example we examine hourly ozone fluxes $(\mathrm{kg} /$ hectare). The spatial domain, $D$, shown in Figure 1 , is a $30 \times 30$ regular spatial grid; the dimensions of each cell on the grid are $36 \mathrm{~km} \times 36 \mathrm{~km}$. The lattice for EPA Models- 3 is a two dimensional grid obtained taking into account the effects of the earth curvature. Models- 3 provides the estimated flux for the middle point of each cell, i.e. $\Delta=36 \mathrm{~km}$, where $\Delta$ is the spacing between observed values. In this example we analyze the spatial temporal structure of a process $Z$, which is the hourly averaged ozone fluxes $(\mathrm{kg} / \mathrm{hectare})$ in June 1996 (June 2-June 4). We have 72 observations over time. Before applying our tests for stationarity and separability we need to remove the spatial-temporal trend.

In order to remove the spatial trend, we calculated at each location the ozone anomaly, that is the corresponding ozone value minus the mean over time (using the 72 observations over time at each location). Figure 2 clearly indicates a diurnal cycle. We removed this temporal trend using a cosine and sine function with a period of 24 hours. The diurnal cycle of the ozone anomalies appeared to be the same everywhere. Figure 3 shows the residuals after the diurnal cycle was removed. We implemented our test to the ozone anomalies (after removing this diurnal cycle).

The estimates, $\hat{f}_{\mathbf{a b}}(\boldsymbol{\omega})$ were obtained using expression (10) in which $W(\alpha)$ is given by (12) with a bandwidth of $2 \pi B$ with $B=1 / 12$, and $g_{\rho}(\mathbf{u})$ is of the form (11) with $\rho=5.5$ units ( 1 unit $=36 \mathrm{~km}$ ), $m=B T$. Thus, in order to obtain approximately uncorrelated estimates, the frequencies $\omega_{j}$ and pairs $\left\{\left(\mathbf{a}_{i}, \mathbf{b}_{i}\right)\right\}_{i=1}^{k}$ should be chosen so that the spacings between the $\omega_{j}$ are at least $\pi / 6$ and the distance between any pairs $\left(\mathbf{a}_{i}, \mathbf{b}_{i}\right)$ and $\left(\mathbf{a}_{j}, \mathbf{b}_{j}\right)$ for $i \neq j$ is at least 5.5 grid cells $(198 \mathrm{~km})$.

The $\omega_{j}$ were chosen as follows $\omega_{j}=\pi j / 17$ with $j=1(3) 16$, corresponding to a uniform spacing of $3 \pi / 17$ (which just exceeds $\pi / 6$ ). We evaluate $\hat{f}_{\mathbf{a b}}(\omega)$ at the following frequencies, $\omega_{1}=\pi / 17, \omega_{2}=4 \pi / 17$, $\omega_{3}=7 \pi / 17, \omega_{4}=10 \pi / 17, \omega_{5}=13 \pi / 17$, and $\omega_{6}=16 \pi / 17$. We consider six pairs, $\left\{\left(\mathbf{a}_{i}, \mathbf{b}_{i}\right)\right\}_{i=1}^{6}$, such that the distance between pairs is at least $216 \mathrm{~km}$ (which just exceeds $\rho$ ) for all $i$, where $\|$.$\| denotes the Euclidean$ distance. Figure 1 shows the locations of $\mathbf{a}_{i}$ for $i=1, \ldots, 6$, which correspond to the sites $A, B, \ldots$, and $F$, respectively. The locations of $\mathbf{b}_{i}$ for $i=1, \ldots, 6$, are other six sites at the same latitude as $A, B, \ldots$, and $F$ but $72 \mathrm{~km}, 144 \mathrm{~km}, 180 \mathrm{~km}, 72 \mathrm{~km}, 144 \mathrm{~km}$, and $180 \mathrm{~km}$ further east, respectively. Table 1 shows the the results of the test for separability, using these 6 pairs. 


\begin{tabular}{|lllll|}
\hline Item & Degrees of freedom & Sum of squares & F Value & $\operatorname{Pr}(\mathrm{F})$ \\
\hline \hline Between spatial points & 5 & 10.4784 & 6.1440 & 0.0007 \\
Between frequencies & 5 & 25.5483 & 14.9802 & 0.0000 \\
Residuals & 25 & 8.5273 & & \\
\hline
\end{tabular}

Table 1. Analysis of variance (all 6 sites).

The "between spatial locations" effect is highly significant ( $\mathrm{p}$-value $<0.01$ ), confirming that there is clear evidence of lack of separability. The coefficient of determination is .81 .

In the next table we study separability in a smaller subregion (the eastern part of our domain) using only pairs $\left(\mathbf{a}_{3}, \mathbf{b}_{3}\right)$ and $\left(\mathbf{a}_{6}, \mathbf{b}_{6}\right)$. Table 2 shows that the "between spatial locations" effect is not significant, suggesting that then there is no evidence of lack of stationarity (in the eastern part of our domain using pairs $\left(\mathbf{a}_{3}, \mathbf{b}_{3}\right)$ and $\left.\left(\mathbf{a}_{6}, \mathbf{b}_{6}\right)\right)$. However, the "between frequencies" effect is highly significant, confirming that the process is nonseparable. The coefficient of determination is .97. Even in a smaller subregion the assumption of separability is still unrealistic (Table 2). The distance between the two components in both pairs is the same, so we could use these two pairs to test for stationarity. It appears that stationarity in that smaller subregion is a reasonable assumption. Figure 4 shows a qqplot of the residuals to study the potential deviation from normality, but clearly normality seems to be a reasonable assumption.

\begin{tabular}{|lllll|}
\hline Item & Degrees of freedom & Sum of squares & F Value & $\operatorname{Pr}(\mathrm{F})$ \\
\hline \hline Between spatial points & 1 & 0.0129 & 0.1872 & 0.6832 \\
Between frequencies & 5 & 11.1377 & 32.1995 & 0.0008 \\
Residuals & 5 & 0.3459 & & \\
\hline
\end{tabular}

Table 2. Analysis of variance (using sites $\mathbf{c}$ and $\mathbf{f}$ ).

Table 3 shows the $\left|\hat{R}_{\mathbf{a b}}(\omega)\right|^{2}$ values at each one of the frequencies $\omega_{j}$ for $j=1, \ldots, 6$ for the pairs $\left(\mathbf{a}_{3}, \mathbf{b}_{3}\right)$ and $\left(\mathbf{a}_{6}, \mathbf{b}_{6}\right) .|R|^{2}$, can be interpreted as a coefficient of correlation: values close to 1 indicate high correlation between the two time series. Both pairs seem to have a very similar coherency function, which supports the assumption of stationarity. However, the coherence is clearly changing with frequency. Thus, it seems that locally it might reasonable to assume stationarity for ozone fields, but separability seems to be unrealistic even for small geographic areas. 


\begin{tabular}{|l|llllll|}
\hline Pairs: & $\omega_{1}$ & $\omega_{2}$ & $\omega_{3}$ & $\omega_{4}$ & $\omega_{5}$ & $\omega_{6}$ \\
\hline \hline$\left(\mathbf{a}_{3}, \mathbf{b}_{3}\right)$ & 0.769 & 0.648 & 0.891 & 0.960 & 0.994 & 0.999 \\
$\left(\mathbf{a}_{6}, \mathbf{b}_{6}\right)$ & 0.931 & 0.660 & 0.840 & 0.978 & 0.994 & 0.998 \\
\hline
\end{tabular}

Table 3. Coherence for pairs $\left(\mathbf{a}_{3}, \mathbf{b}_{3}\right)$ and $\left(\mathbf{a}_{6}, \mathbf{b}_{6}\right)$ at six frequencies.

\section{Discussion and power of the test}

We have presented in this paper a new method to test for separability and stationarity of spatial-temporal processes. The mechanics of the test proposed here can be reduced to a simple two-factor ANOVA procedure.

Several simulation studies were conducted to estimate the power of our test to detect deviations from separability when the underlying covariance was not separable. We simulated 500 versions of a spatial temporal Gaussian process on the same grid as in the air quality application shown here, with a nonseparable covariance. The covariance function used was a 3-dimensional exponential covariance:

$$
C(x, y, t)=\sigma_{0}^{2} I_{\{(x, y, t)=0\}}(x, y, t)+\sigma^{2} \exp \left\{-\left(x^{2}+y^{2}+t^{2}\right) / \alpha\right\}^{1 / 2}
$$

where $I$ is an indicator function that takes the value 1 when $\{(x, y, t)=\mathbf{0}\}$, and it is zero otherwise, the value of the nugget parameter was $\sigma_{0}^{2}=.01$, the range was $\alpha=2$ and the partial sill was $\sigma^{2}=1$. We used the same pairs and frequencies as in our application. The estimated probability of rejecting the null hypothesis of separability when the underlying process was separable (the product of two exponentials with nugget of .01 , range of 2 and partial sill of 1) was .08 (Type I error). Out of the 500 simulated fields 455 (91\%) rejected the null hypothesis of separability (for a level $\alpha=.05$ test). We repeated this simulation 20 times, and we consistently got similar results with an average of .9 power.

The approach proposed here is designed to test the separability of the covariance function when the mean of the process has been removed. If the mean of the process is a function of space and time the test still seemed to be powerful enough to detect the nonseparability of the underlying process, even if we do not remove the mean surface. We conducted a simulation study as above but with a nonseparable mean function that was a quadratic polynomial in space-time. The power was .99 to detect lack of separability when the underlying covariance was nonseparable, but it also rejected $80 \%$ of the time separability (due to 
the nonseparable mean) when the covariance was separable.

Our test of separability is based on the spectral methods and theory for nonseparable processes introduced in this paper. We propose a nonparametric estimate of the spectral density of the process. This spectral estimate, $\hat{f}_{\mathbf{a b}}(\omega)$, is a function of two filters; $\left\{g_{\rho}(\mathbf{s})\right\}$ and $\{W(\omega)\}$. These two filters are needed to guarantee the independence of our estimated spectrum at locations and frequencies that are sufficiently far apart with respect to some bandwidth parameters (so we can use ANOVA). Thus, before $\hat{f}_{\text {ab }}(\omega)$ can be evaluated we have to choose the form of the two filters. Generally, $\left\{g_{\rho}(\mathbf{s})\right\}$ will be chosen from the standard collection of "windows" and will involved a parameter $\rho$. Similarly, we may choose $\{W(\omega)\}$ from the same collection of windows with a parameter $B$. Suppose now that we have chosen the mathematical forms of $\left\{g_{\rho}(\mathbf{s})\right\}$ and $\{W(\omega)\}$. The problem arises as to how we should choose the parameters $B$ and $\rho$ so that the estimated $\hat{f}_{\text {ab }}(\omega)$ possesses certain required properties. If there are no requirements for specified degrees of resolvability in either domain, we may choose $B$ and $\rho$ so as to jointly minimize the relative mean-square error of $\hat{f}_{\text {ab }}(\omega)$. We could allow the parameters $B$ and $\rho$ to be both space and frequency selective. The asymptotic results obtained in this paper (Theorem 1) provide an approximation for the mean squared error $(\mathrm{MSE})$ of $\hat{f}_{\mathbf{a b}}(\omega)$. We suggest using a plug-in approach replacing $f_{\mathbf{a b}}(\omega)$ with $\hat{f}_{\mathbf{a b}}(\omega)$ in the expression for the MSE, to obtain the values of $B$ and $\rho$ that minimize the relative MSE of $\hat{f}_{\mathbf{a b}}(\omega)$.

As part of our future work we are developing a formal test to study directional symmetry. This test would be another side-effect of the test for separability proposed here, by studying the significance of the interaction term in the two-factor analysis of variance model in Section 4.

\section{Acknowledgments.}

The author would like to thank Dr. R. Martin, University of Sheffield, for his helpful and insightful comments. 


\section{APPENDIX}

For the proof of Theorem 1 we will need the following lemma.

Lemma 1: Let $\mathbf{Z}^{(T)}, T=1,2, \ldots$ be a sequence of s vector-valued complex random variables, Assume all the cumulants $\left[Z_{1}^{(T)}, \bar{Z}_{1}^{(T)}, \ldots, Z_{s}^{(T)}, \bar{Z}_{s}^{(T)}\right]$ exist and tend to the cumulants of a variate $\left[Z_{1}, \bar{Z}_{1}, \ldots, Z_{s}, \bar{Z}_{s}\right]$ that is determined by its moments. Then $\mathbf{Z}^{(T)}$ converges in distribution to a variate having components $Z_{1}, \ldots, Z_{s}$

Proof of Lemma 1: All the convergent subsequences of the sequence of cdf's of $\mathbf{Z}^{(T)}$ converge in distribution to variables with the given moments. Since we are assuming that there is only one cdf with those moments then $\mathbf{Z}^{(T)}$ converges in distribution to a variate having components $Z_{1}, \ldots, Z_{s}$.

Proof of Theorem 1 (i): The first expression in (13) is obtained by writing $\hat{f}$ (defined in (10)) in terms of the periodogram (9), and then calculating the expression for the expected value of a second-order periodogram (Brillinger (1981), page 122). We approximate the sum by an integral, then we get

$$
\begin{aligned}
E\left(\hat{f}_{\mathbf{a b}}(\omega)\right) & =\frac{2 \pi}{T} \sum_{r=1}^{T-1} W^{(T)}\left(\omega-\frac{2 \pi r}{T}\right) \tilde{f}_{\mathbf{a b}}\left(\frac{2 \pi r}{T}\right)+O\left(T^{-1}\right) \\
& =\int_{-\pi}^{\pi} W^{(T)}(\omega-\alpha) \tilde{f}_{\mathbf{a b}}(\alpha) d \alpha+O\left(B^{-1} T^{-1}\right) \\
& =\int_{-\infty}^{\infty} B^{-1} W\left(B^{-1}[\omega-\alpha]\right) \tilde{f}_{\mathbf{a b}}(\alpha) d \alpha+O\left(B^{-1} T^{-1}\right)
\end{aligned}
$$

that leads to the final expression in (13).

Proof of Theorem 1 (ii): We obtain that the covariance is of the form

$$
\begin{aligned}
\operatorname{cov}\left\{\hat{f}_{\mathbf{a}_{1}, \mathbf{b}_{1}}(\lambda), \hat{f}_{\mathbf{a}_{2}, \mathbf{b}_{2}}(\omega)\right\} & =\left(\frac{2 \pi}{T}\right)^{2}\left[\sum_{r=1}^{T-1} W^{(T)}\left(\lambda-\frac{2 \pi r}{T}\right) W^{(T)}\left(\omega-\frac{2 \pi r}{T}\right)\left[\tilde{f}_{\mathbf{a}_{1} \mathbf{a}_{2}}\left(\frac{2 \pi r}{T}\right) * \tilde{f}_{\mathbf{b}_{1} \mathbf{b}_{2}}\left(-\frac{2 \pi r}{T}\right)\right]\right. \\
& \left.+\sum_{r=1}^{T-1} W^{(T)}\left(\lambda-\frac{2 \pi r}{T}\right) W^{(T)}\left(\omega+\frac{2 \pi r}{T}\right)\left[\tilde{f}_{\mathbf{a}_{1} \mathbf{b}_{2}}\left(\frac{2 \pi r}{T}\right) * \tilde{f}_{\mathbf{b}_{1} \mathbf{a}_{2}}\left(-\frac{2 \pi r}{T}\right)\right]\right] \\
& +O\left(T^{-1}\right),
\end{aligned}
$$

expression (14) follows by by replacing the sum by an integral (Polya and Szergö (1925) page 37).

\section{Proof of Theorem 1 (iii):}

The proof of (iii) is a corollary of Theorem 1 (ii) when $B T \rightarrow \infty$ as $T \rightarrow \infty$. 
Proof of Theorem 1 (iv): We have already studied the asymptotic first and second-order moment of

the spectrum estimate $\hat{f}$. We prove now the asymptotic joint normality by showing that all standardized joint cumulants of order greater than 2 tend to 0 as $T \rightarrow \infty$ under the indicated conditions.

Using a similar approach to the one in Brillinger (1981) page 437) we obtain that the cumulant

$$
\operatorname{cum}\left\{(B T)^{1 / 2} \hat{f}_{\mathbf{a}_{1} \mathbf{b}_{1}}\left( \pm \omega_{1}\right), \ldots,(B T)^{1 / 2} \hat{f}_{\mathbf{a}_{J} \mathbf{b}_{J}}\left( \pm \omega_{J}\right)\right\}
$$

is of order $B^{-J / 2+1} T^{-J / 2+1}$ so it goes to 0 as $T \rightarrow \infty$ for $J>2$. Thus, the asymptotic normality follows now from Lemma 1.

We have next the proofs of Theorem 2 and 3 that follow directly from Theorem 1. Because once we have the asymptotic properties of the estimated spectrum the asymptotic distribution of the coherency and its stabilizing transformation follow straightforward from the delta-method.

\section{Proof of Theorem 2:}

We use a Taylor series expansion,

$$
\begin{aligned}
\hat{R}_{\mathbf{a b}}(\omega) & =\tilde{R}_{\mathbf{a b}}(\omega)+\left[\left\{\hat{f}_{\mathbf{a b}}(\omega)-\tilde{f}_{\mathbf{a b}}(\omega)\right\}-\frac{1}{2} \tilde{f}_{\mathbf{a a}}(\omega)^{-1}\left\{\hat{f}_{\mathbf{a a}}(\omega)-\tilde{f}_{\mathbf{a a}}(\omega)\right\}\right. \\
& \left.-\frac{1}{2} \tilde{f}_{\mathbf{b b}}(\omega)^{-1}\left\{\hat{f}_{\mathbf{b b}}(\omega)-\tilde{f}_{\mathbf{b b}}(\omega)\right\}\right] /\left[\tilde{f}_{\mathbf{a a}}(\omega) \tilde{f}_{\mathbf{b b}}(\omega)\right]^{2}+\ldots
\end{aligned}
$$

to derive the mean and covariance of $\hat{R}$ from the mean and covariance of $\hat{f}$ obtained in Theorem (i) and (ii) respectively. The asymptotic normality follows from Theorem 1 (iv) and the delta-method.

Proof of Corollary: The hyperbolic inverse tangent is the variance stabilizing transformation for $\hat{R}$. The mean, covariance and asymptotic normality follows from Theorem 1 and the delta-method.

\section{References}

Brillinger, D. R. (1981). Time Series: Data Analysis and Theory. Expanded edition. Holden-Day, Inc, San Francisco.

Bose, S. and Steinhardt, A. O. (1996). Invariant tests for spatial stationarity using covariance structure, IEEE Transactions on Signal Processing, 44, 1523-1533. 
Brown, P., Karesen, K. and Tonellato, G.O.R.S. (2000). Blur-generated nonseparable space-time models. Journal of the Royal Statistical Society, Series B, 62, 847-860.

Cressie, N. and Huang, H.-C. (1999). Classes of nonseparable, spatio-temporal stationary covariance functions. Journal of the American Statistical Association, 94, 1330-1340.

Ephraty, A., Tabrikian, J. and Messer, H. (2001). Underwater source detection using a spatial stationary test, Journal of the Acoustical Society of America, 109, 1053-1063.

Fuentes, M. (2001). A new high frequency kriging approach for nonstationary environmental processes. Envirometrics, 12, 469-483.

Fuentes, M. (2002). Spectral methods for nonstationary spatial processes. Biometrika, 89 197-210.

Fuentes, M. (2003). A formal test for nonstationarity of spatial stochastic processes. Journal of Multivariate Analysis, to appear.

Guo, J. H. and Billard, L. (1998). Some inference results for causal autoregressive processes on a plane. J. Time Ser. An., 19 681-691.

Haas, T. C. (1998). Statistical Assessment of Spatio-Temporal Pollutant Trends and Meteorological Transport Models, Atmospheric Environment, 32(11): 1857-1864.

Kendall M. G., and Stuart A. (1966). The advanced theory of statistics. Volume 3: Design and analysis and time-series. Griffin, London.

Lu, N., Zimmerman, D. L. (2002). Testing for directional symmetry using the periodogram. Res. Rep. \# 317, Department of Statistics and Actuarial Science, University of Iowa.

Mann, H. B., and Wald, A. (1943). On Stochastic Limit and Order Relationships, Annals of Mathematical Statistics, 14, 217-226.

Mitchell, M. (2002). Testing Separability of Covariances for Space-Time Processes. Ph.D. Thesis at the Statistics Department, North Carolina State University.

Mitchell, M., Genton, M. G., Gumpertz, M. (2002) A likelihood ratio test for separability of covariances, manuscript under review. (http://www4.ncsu.edu:8030/ mwmitch2/Biom.pdf)

Polya, G. and Szergö, G. (1925). Aufgaben and Lehrsätze aus der Analysis I. Berlin: Springer.

Priestley, M. B. and Rao, T. S. (1969). A test for non-stationarity of time-series. Journal of the Royal 
Statistical Society, Series B, 31, 140-149.

Rao, C. R. (1973).Linear Statistical Inference and Its Applications. Second Edition. Wiley, New York.

Scaccie, L., Martine, R. J. (2003). Testing axial symmetry and separability of lattice processes. Journal of Statistical Planning and Inference, to appear.

Shitan, M. and Brockwell, P. (1995). An asymptotic test for separability of a spatial autoregressive model. Communications in Statistics - Theory and Methods, 24, 2027-2040. 


\section{Ozone deposition flux, June 21996}

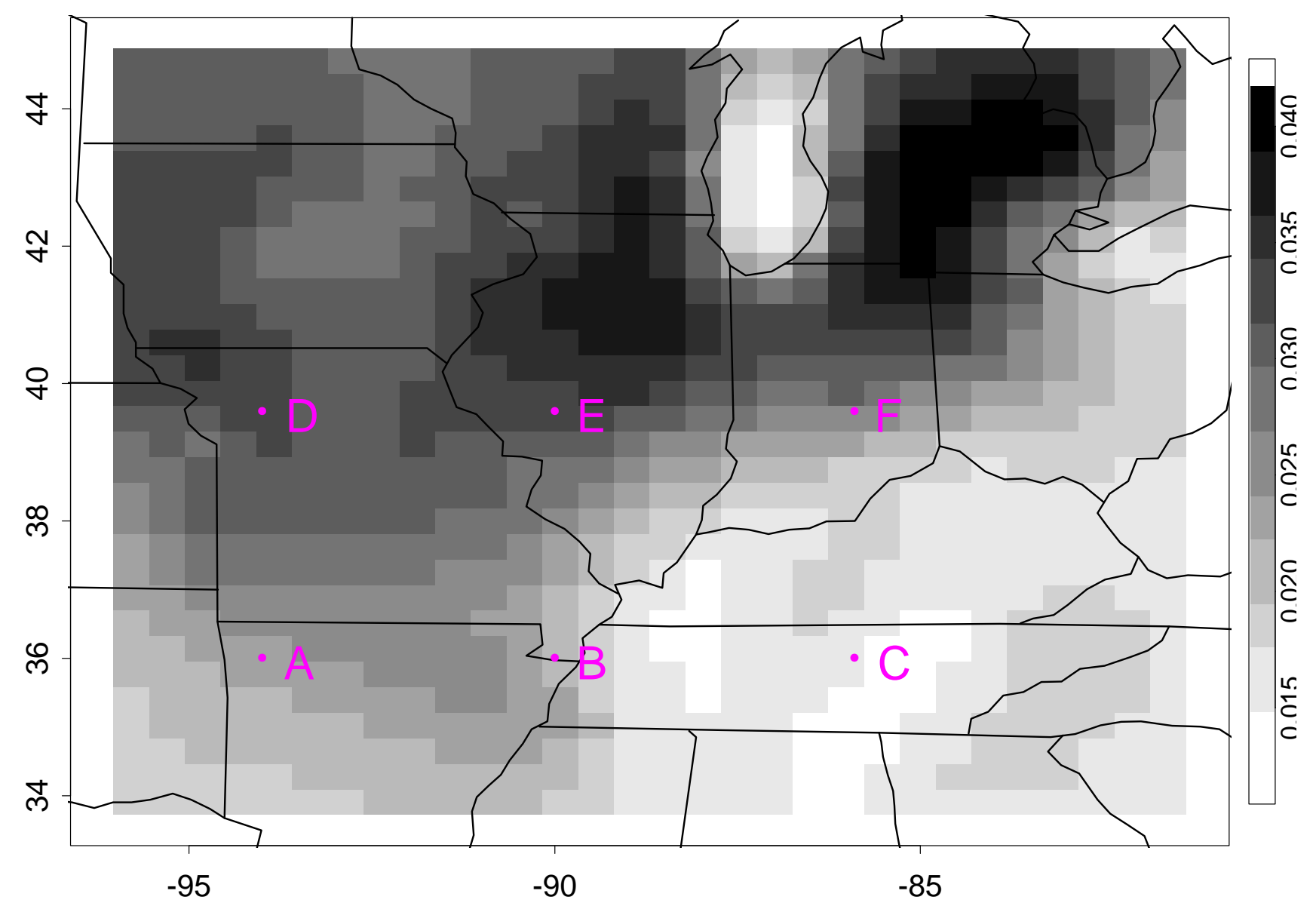

Figure 1: This graph shows the ozone deposition flux $(\mathrm{kg} /$ hectare) in a region in the Midwest. The values in this graph are the output of the EPA regional scale air quality model (Models-3) on June 2 at 12pm central time. 


\section{Time series plots of Ozone flux}

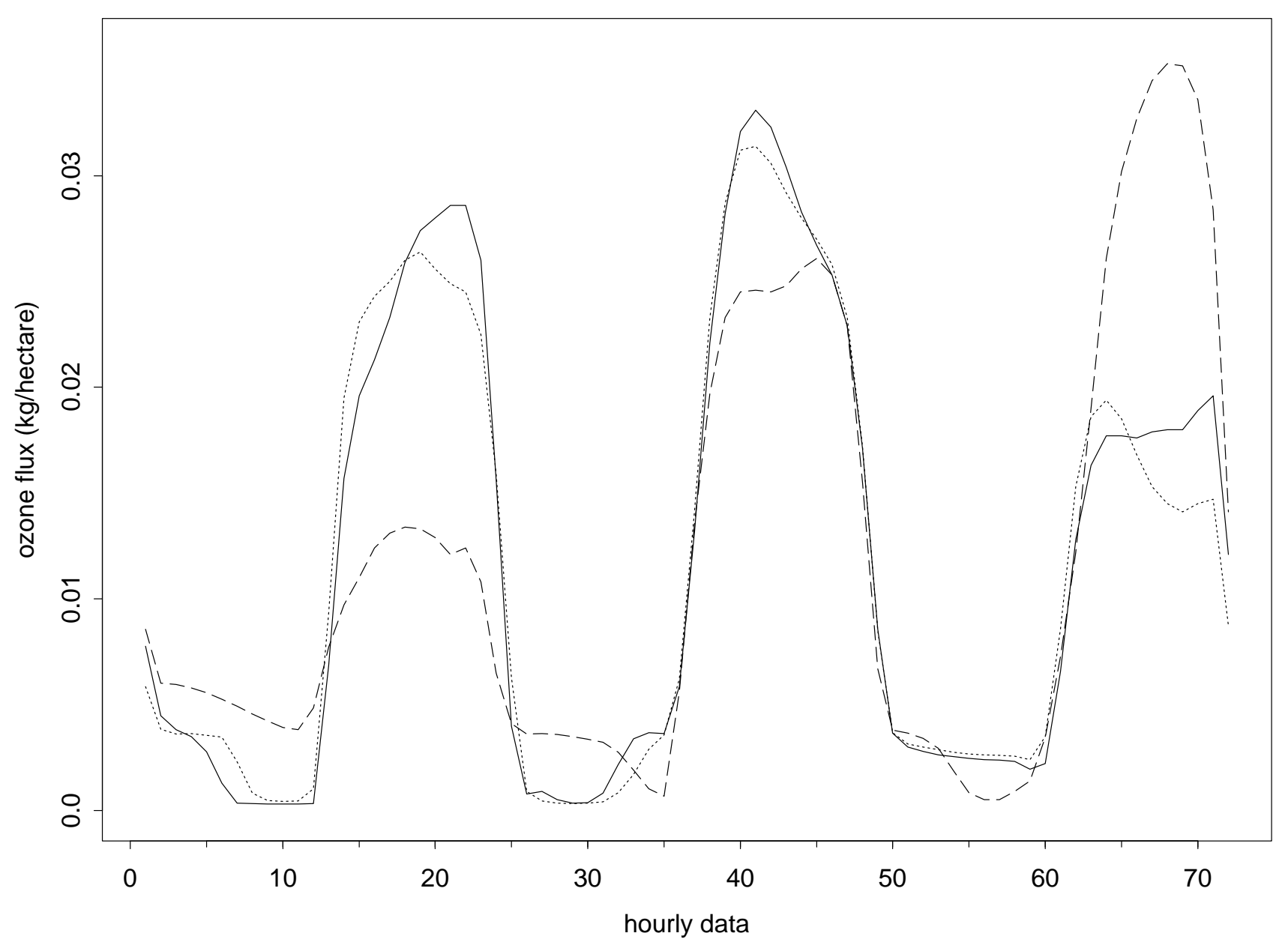

Figure 2: The time series in this graph show hourly ozone deposition fluxes (kg/hectare) for June 2, June 3, and June 4, 1996, at 3 locations with the same latitude but with different longitude coordinates. The locations are sites A (solid line), and C (dashed line), and a site (dotted line) $72 \mathrm{~km}$ east of location A. The ozone values are output from the numerical air quality models (Models-3). 


\section{Time series plots of Residuals}

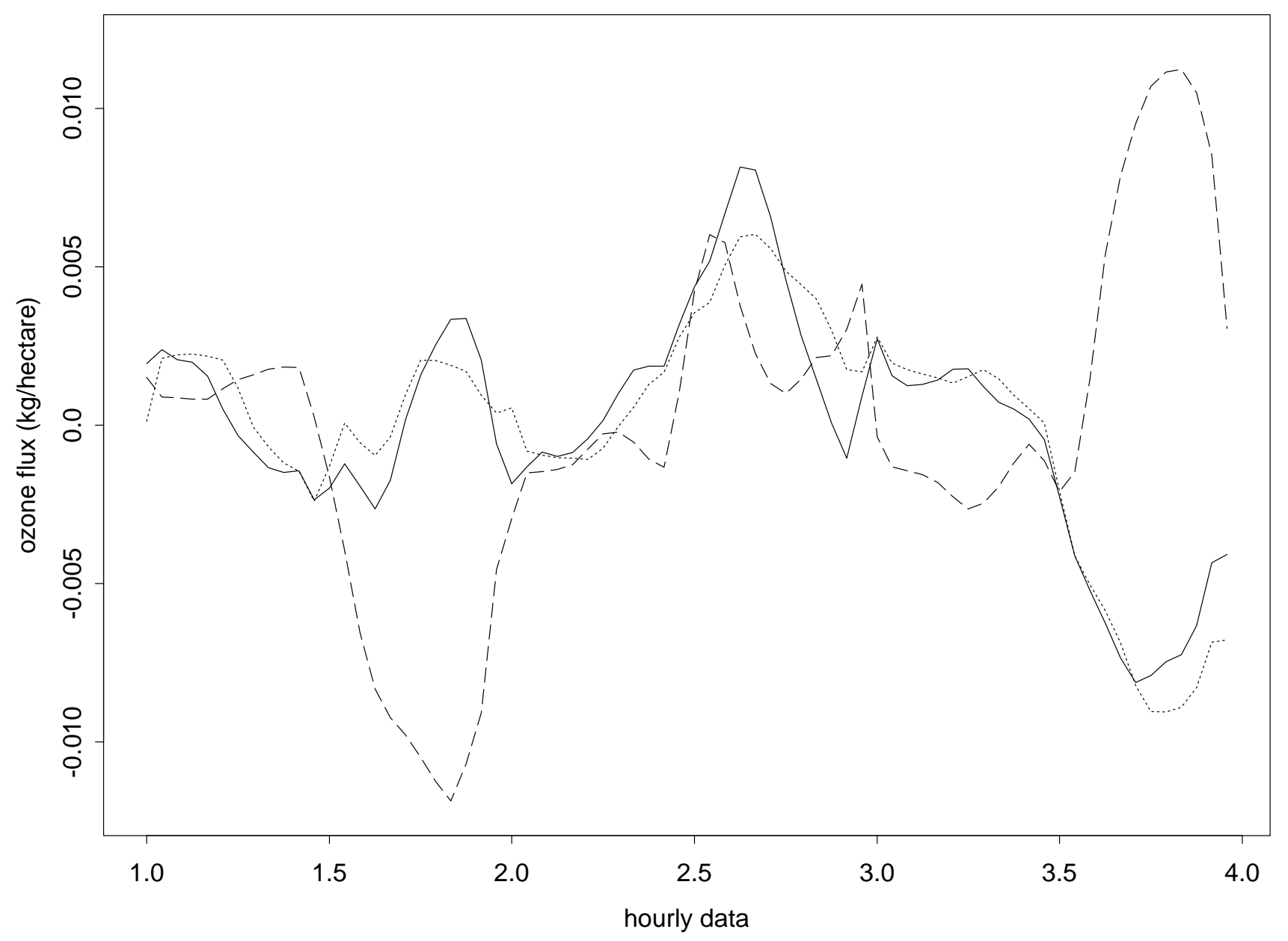

Figure 3: The time series in this graph show the ozone anomalies (ozone fluxes minus the mean value at each location) for June 2, June 3, and June 4, 1996, after removing the diurnal cycles at 3 locations with the same latitude but with different longitude coordinates. The locations are sites A (solid line), and C (dashed line), and a site (dotted line) $72 \mathrm{~km}$ east of location A. 


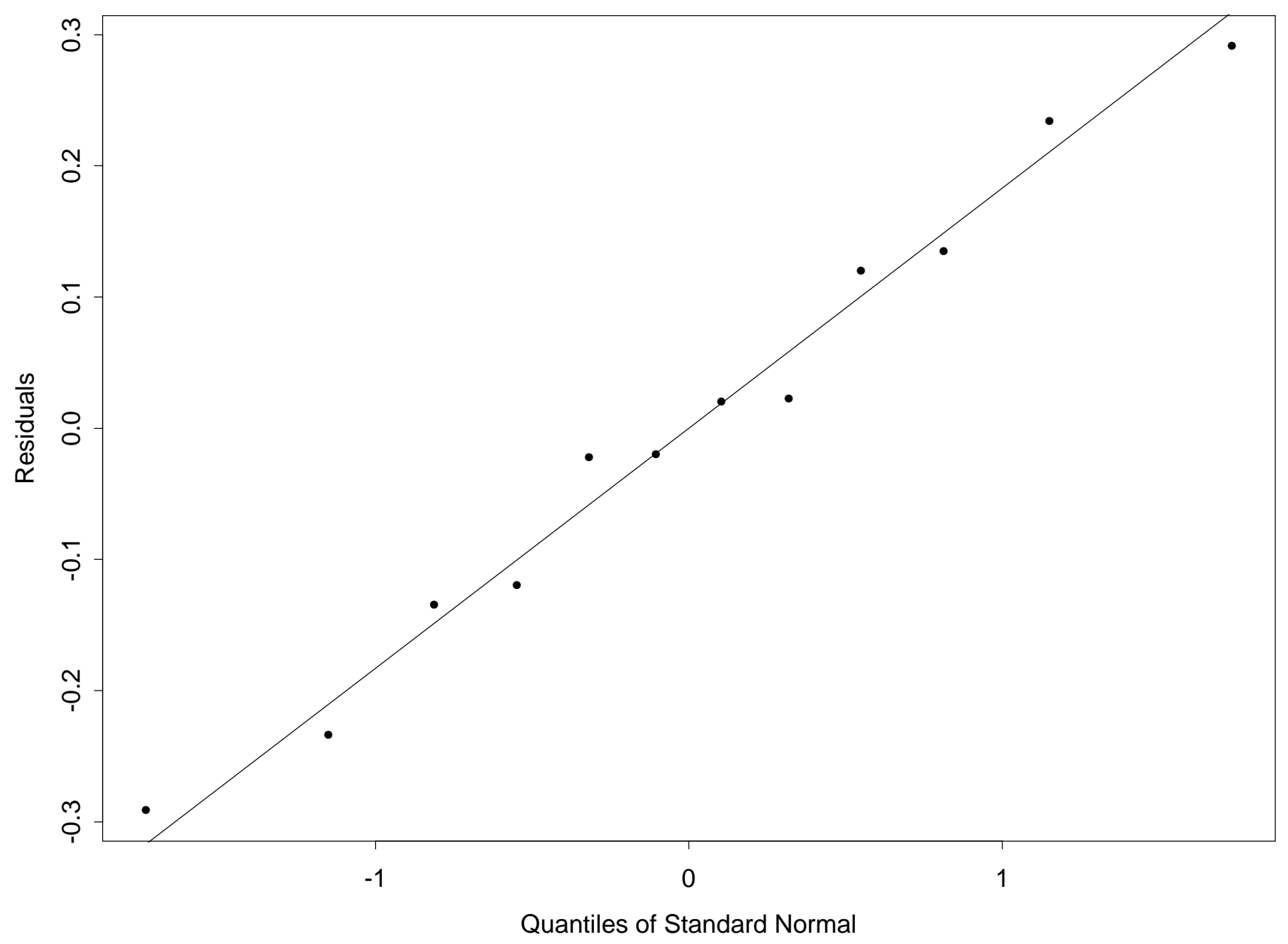

Figure 4: This graph shows a QQplot to assess whether the residuals from the analysis of ANOVA in Table 2 have a normal distribution. 